\title{
The Insider on the Outside: A Novel System for the Detection of Information Leakers in Social Networks
}

\begin{abstract}
Confidential information is all too easily leaked by naive users posting comments. In this paper we introduce DUIL, a system for Detecting Unintentional Information Leakers. The value of DUIL is in its ability to detect those responsible for information leakage that occurs through comments posted on news articles in a public environment, when those articles have withheld material nonpublic information. DUIL is comprised of several artifacts, each designed to analyse a different aspect of this challenge: the information, the user(s) who posted the information, and the user(s) who may be involved in the dissemination of information. We present a design science analysis of DUIL as an information system artifact comprised of social, information, and technology artifacts. We demonstrate the performance of DUIL on real data crawled from several Facebook news pages spanning two years of news articles.
\end{abstract}

keyword: Cybersecurity; Online Social Networks; Information Leakers; Sensitive Information; Threat Detection; Design Science Research.

\section{Introduction}

In the field of information security, an insider threat is defined as 'the organisational member who is a trusted agent inside the firewall' (Im and Baskerville 2005). Information security specialists try to protect against information leakage by detecting and blocking insider threats brought on by actors who are, by definition, organisational insiders. Warkentin 
and Willison (2009) describe the greatest insider threat as the 'employee or other constituent with a valid user-name and password (who) regularly interacts with the information assets of the organization' (p. 102). Bishop and Gates (2008) extend the definition of insider threat to those with access irrespective of an inside affiliation through either (a) violation of a security policy using legitimate access; or (b) violation of an access control policy by obtaining unauthorised access. Studying areas such as user accountability to address access violations [ADD VANCE REF] helps deal with threats from users known to the organisation, again with a focus on access.

Rather than focusing on access, the field of finance provides a more information-centric definition of an insider, being anyone who is privy to information that has not been released to the general public. This is based on the concept of information asymmetry (Huddart and Ke 2007), where an insider is deemed to be anyone who has an information advantage over other market participants. In that context, the goal is to detect or prevent insider trading which is the practice of trading in the securities markets by those in possession of material nonpublic information (Karsch 1984). Following this definition, should a senior manager in a public company share unreleased material information about company performance with his neighbour, and as a result that neighbour trades in the public markets, the neighbour has committed an act of insider trading and is treated as a de-facto insider (Strudler and Orts 1999).

Following the above definition, when an organisation has information that it intends to remain hidden, secret, or censored, anyone who possesses that information, regardless of organisational affiliation or access control, becomes an insider. What was once considered an organisational problem focused on identifying threats emanating from those connected to the organisation, has quickly become a broader problem in which any member of society at large may have access to material nonpublic content generated by users through a social network. We harness user-generated content (UGC, see Agichtein et al. (2008)) to identify internal and external information leakers. UGC is generally characterised by (1) a broad and 
unrestricted user base, (2) user identifying information, (3) user social network, and (4) the contributed content. Example of UGC types include blogs, video channels such as YouTube and micro-blogs such as Twitter.

In this work we focus on comments to articles containing incomplete information, where the hidden information is held by insiders. Our demonstrations present censored military articles in which the identity of personnel is withheld. Insiders - particularly those in the know often outside of the military organisation, can share their private information through comments. Similar forms of identity protection can be found in different domains and cultures, such as non-release of rape victim names in trial reporting or nondisclosure of customer names in corporate announcements as discussed in Yahav, Schwartz, and Silverman (2014). We suggest that with the present information environment of social networks, cyber-security against an insider threat must consider the external insider - the insider on the outside.

The cyber environment is becoming increasingly complex. The field of intelligence gathering is concerned with covert operations, attempts to crack and access protected information assets and supporting infrastructures, and the collection and analysis of Open Source INTelligence (OSINT) (Burattin, Cascavilla, and Conti 2015; Kandias et al. 2013). In the intelligence community, the term 'open' refers to overt publicly available sources - as opposed to covert or clandestine sources. Hence, OSINT approaches aim at extracting knowledge from publicly available sources (Kandias et al. 2013), which includes on-line comments. Claudio (2009) discusses how both social network analysis and visualisation are fundamental to cyber deterrence strategy, pointing to the growing need to develop advanced detection systems, incorporating linguistic cues (Zhou et al. 2004) and visualisations to effectively identify OSINT social network threats. Earlier results reported in Cascavilla et al. (2015) describe the information leakage problem and detection. Expanding our previous work, the present work describes the full system view and functionality.

An abundance of information is exchanged in the commenting environments of news articles. In this work, we present DUIL, a system for Detecting Unintentional Information 
Leakers. This novel system for information leaker detection is initially applied to a set of censored news articles. DUIL is comprised of a loosely-coupled set of artifacts that implement a multi-stage leaker detection process which can be generalised for the detection of leakers in other information environments by replacing certain artifacts in the system, so long as the UGC characteristics remain. The study and analysis of this type of system naturally points us in the direction of design science research (DSR) (Hevner et al. 2004; Hevner and Chatterjee 2010).

Our work presents two practical contributions. First, our work raises the important question of leakers, who unintentionally uncover hidden information via UGC, specifically, comments to on-line news in the public sphere. Second, we present a novel end-to-end system that is designed to detect such information leakers, along with their social network. We present a modular architecture system that can be tuned to any (user-) given news context, as long as the data analysed is UGC, as we demonstrate through a collected dataset of censored news articles.

Our work also presents two secondary theoretical contributions to design science research that are distinct from the novel information system itself. The first theoretical contribution relates to use of the Lee, Thomas, and Baskerville (2015) IS artifact framework. We show how this framework, when applied to a complex multi-artifact information system, improves expressiveness and clarity in presenting design science research (DSR). The second theoretical contribution relates to the Gregor and Hevner (2013) DSR Knowledge Contribution Framework. We draw upon the framework of Lee, Thomas, and Baskerville (2015) to present enhancements to the DSR Knowledge Classification framework, extending its applicability to complex multi-artifact information systems and adding expressiveness to the original 4-quadrant classification. We believe that the above will contribute to the consistency of DSR reporting.

This introductory section has focused on motivating the research problem, the detection of unintentional information leakers in social networks. In our Literature Review in the next 
section, we discuss contributions addressing related research problems and elaborate on the research gap. A formal introduction of our chosen methodology in the third section is followed by the fourth section on the Study Scope which covers problem identification, solution objectives, and the fifth section with a comprehensive presentation of system design. We then demonstrate and evaluate in the sixth section the operation and effectiveness of our DUIL system through a series of experiments designed around the current UGC context of news commenting. Our discussion in the final section includes use of the DSR Knowledge Contribution Framework to provide insights into the knowledge contribution of DUIL's design, and reflects upon how the IS artifact framework informs the DSR process.

\section{Literature Review}

\section{Design Science Research}

Iivari (2014) distinguishes between two different DSR strategies dominating the information systems literature. One strategy, in the tradition of Markus, Majchrzak, and Gasser (2002) and Sein et al. (2011) which could be called client-centric or organisation-centric DSR, begins with an attempt to solve a specific client's existing problem and progresses towards a generalisation useful in other contexts. Iivari contrasts this with a 'proof of concept' approach in which a system is constructed to address a general problem and then instantiated as a test of the design theory. We have chosen to follow the 'proof of concept' path in the context of a multidisciplinary international collaboration as advocated by Nunamaker et al. (2017). This is similar to the approach taken by Twyman et al. (2014) who also address aspects of information leakage in their work. These more recent approaches are combined with the tradition of Hevner et al. (2004), Peffers et al. (2007), and the formalisations provided in Gregor and Hevner (2013). 


\section{Information System Artifacts}

There has been considerable debate around the question of artifacts and their centrality to DSR. Much of DSR work describes information technology (IT) artifacts, which Orlikowski and Iacono (2001) and Orlikowski and Iacono (2006) define as 'bundles of material and cultural properties packaged in some socially-recognisable form such as hardware and/or software'. However not all agree that the 'bundled' IT artifact should be the focal point of information systems research in general and DSR in particular. Lyytinen and King (2004) note that IT artifacts do not deliver value in their own right and must be viewed in the context of a system. Schwartz (2014) advocates the decomposition of IT artifacts into several distinct yet interconnected artifacts. Most recently, Lee, Thomas, and Baskerville (2015) suggest a multi-artifact view when approaching DSR, arguing that the IT artifact is just one element within a broader Information systems artifact, which should be viewed as a construct incorporating information, social, and technology artifacts - and must be addressed as such in design science research.

We have chosen to augment our presentation by addressing the relatively new framework of IS artifacts articulated by Lee, Thomas, and Baskerville (2015). As we will see, this approach is very well suited to the task and in using it to frame our work we believe we contribute to increased understanding and potential use of the framework.

Lee, Thomas, and Baskerville (2015) define a framework, which is comprised of three major elements as follows:

1. A Social artifact - an artifact embodying relationships or interactions among multiple individuals;

2. An Information artifact - an instantiation of information produced by a human participant either directly (as their own creative output) or indirectly (through an individuals invocation of a software program or other automated information production process);

3. A Technology artifact - a human-created tool used to solve a human-defined or per- 
ceived problem.

All three interact within a broader systems framework achieving a results that is greater than the sum of its parts, comprising the IS artifact.

\section{Methodological Approach}

This study follows the established approach to design science research as applied by Hevner et al. (2004), Hevner and Chatterjee (2010), and Peffers et al. (2007), resulting in a proof of concept. This approach consists of five stages: 1) problem identification, in which we define the scope of our study: detecting information leakers via commenting to on-line news; 2) going through the solution objectives, that is a set of expectations from the system and its design; 3) artifact design, that is the design of the system, followed by 4) demonstration with real data. The fifth stage is the system evaluation according to the objectives set in the second stage.

The DUIL system presented in this study is the result of integrating a series of independently developed and tested artifacts that were adjusted for the purpose of leaker detection. Each distinct artifact addresses a key aspect of the overall system solution. The first three artifacts, including two information artifacts and one social artifact, evolved from a study of the nature of leakage through UGC, specifically, comments. The latter two are technology artifacts which were developed in the context of uncovering hidden network relationships that reveals the potential scope of the leak. The combination and integration of these five artifacts led to the end-to-end leaker detection information system that we present.

In what follows we describe our study scope: disclosing material nonpublic information in the form of comments to news articles in a given context, which occurs as a direct result of social network structures.

\section{Study Scope}


DUIL is designed to detect users who disclose material nonpublic information through User Generated Content in social media (Agichtein et al. 2008). UGC in characterised by four main components,(1) a broad and unrestricted user base, (2) user or personally identifying information, (3) user social network, and (4) the contributed content.

The release of material nonpublic information can occur either maliciously or unintentionally, through discussions in On-line Social Networks (OSN). It is largely common in articles published by news pages, in which information is withheld, hidden, or censored, only to be uncovered by a commenter. The challenges presented by information leaked through commenting on news articles occur in many different contexts including the identity of military personnel, minor victims, minor perpetrators, rape victims, witnesses and others whose identity is considered information to be withheld from the public, as documented in BlindedForReview2 (2015).

Our solution objectives centre around creating a holistic system to detect information leakers in social networks with an initial focus on Facebook (FB) commenters. While the social network stands at the centre of activities relevant to our work, the types and formats of information that inhabit OSN are myriad. For that reason, modularity becomes our first key objective enabling the use of different information artifacts to capture and analyse different OSN information sources, with an initial focus on news articles and comments.

Automation and accuracy are two closely linked objectives. The vast quantities of information to be processed and the accuracy of per-module results required to contain a security breach create this necessity.

Our third objective is visualisation of leakers' social networks. The detection and identification of information leakers is far from an exact science, and the ability to provide system operators with visualisations of leakers' social network - the extent of the potential leak, is crucial to enabling quick situation assessment and response. 


\section{System Design}

The system is designed to identify information leakers through commenting to on-line news, and provide the system user with network visualisation that presents the direct and indirect relationships between the leakers. The architecture of the system resembles a SwissCheese model, a common model in the risk analysis and management field (Reason 1990), in which at each level non-relevant information is filtered out, and the remaining data are passed to the next module. In our scenario, 'relevant information' refers to comments that disclose knowledge and relationships that may lead to uncovering hidden information not released in the news article, and 'non-relevant information' refers to comments that do not indicate such disclosure. We denote these comments as leak enabling and non-leak enabling respectively. The output of the final module of DUIL is a network visualisation of the relationship between the most relevant commenters.

The system consists of five loosely-coupled modules, corresponding to five artifacts, each responsible for a key part of leak detection and leaker identification:

Module 1 - Articles of Interest (AoI): An information artifact that identifies news articles in a given context. The current implementation detects articles in which personnel names are censored. This results in the creation of an articles of interest dataset for further analysis. It should be noted that in real-time systems, in the specific case in which an official censor is releasing news items, this phase can be replaced with expert input such that AoIs are flagged by the page administrator upon posting by the news agency.

Module 2 - Comments of Interest (CoI): This information artifact focuses on comment analysis. The goal of the module is the identification of news article comment discourse in which the commenters exhibit knowledge of sensitive information not released in the article, hence leak-enabling. This module results in the creation of a comments of interest set, which is passed to the next module. Here too there are multiple approaches to generating this information and the contribution of this artifact to the information system is not in a specific technological approach to comment filtering, but the essential provision 
of information.

Module 3 - Users of Interest (UoI): This social artifact shifts from comments to commenters, and their public user-profile. The objective is to filter out users with close-to-zero probability of being leak-enabling. The remainder set of commenters and their comments is passed to the next module. As we detail below, social network analysis of the users, their characteristics and interactions are a core part of this artifact's contribution, fitting well the description of a social artifact.

Module 4 - EgoNet: Using additional publicly available information, this technology artifact analyses relevant commenters' egocentric networks to enable the detection of implicit relationships between commenters, who are mutual friends. This network of relationships potentially holds additional information that is related to the hidden content, as well as the extent of the information leakage incident. Created specifically as a technological tool for this purpose moves EgoNet clearly into the category of technology artifact.

Module 5 - Viz: The Viz module is a technology artifact that presents a visualisation of leakers' merged social egocentric networks, received as output in module 4. The visualisation provides the system user with a tool to quickly identify the potential risk level of the leak. As a technology artifact it can be easily re-purposed to visualise networks for other types of information systems, but its technological capabilities as a tool remain intact.

This information system design provides for future plans in which different social media sources are analysed to identify the sets of articles of interest, comments of interest and users of interest based on changing criteria and cyber-security needs - necessitating a swap of information and social artifacts.

A detailed description of each module is provided next.

\section{Module 1: AoI}

Given the large set of available FB news pages, the aim of the AoI module is to screen all news posts and generate a database of articles that evolve around a given context: Articles of Interest; along with the full set of comments that follows them, and the users (commenters) 
who posted them. The set of FB news pages, and screening query via regular expressions, are defined by the system user. The output of module AoI is a database, composed of three main tables as follows:

Posts table: a set of Articles of Interest. For each AoI we collect the source (NewsPage); the date; and the content (text).

Comments table: a set of comments that follows the articles. For each comment we store the post identification; the commenter identification (the id of the user that wrote the comment); in reply to comment indicator, if the comment was part of a thread; its date; and its content (text).

Commenters table: a set of users who commented on the post. To reduce system complexity, we do not collect information on all users. We later collect information on-the-spot on Users of Interest (UoI), in modules 3 and 4.

\section{Module 2: CoI}

The CoI (Comments of Interest) Module classifies comments into comments of interest (leak enabling comments) or non interest (non leak enabling). The module contains two steps: (1) an initialisation step, in which multiple classifiers are trained and tested on previously annotated comments; and (2) a classifying step: where comments are classified into the two classes of using the best performing classifier from step (1).

In the initialisation step, a subset of the data is first split into training and evaluation sets. Domain experts are then asked to label the comments as 'leak enabling' or 'non leak enabling' classes. Note that manual classification is only done once, yet is essential for the construction of a meaningful and accurate classifier. A data-driven approach is followed to learn expert labelling. Here, multiple classifier are constructed and trained. Each classifier utilises all or part of the comments' characteristics (e.g., popularity, order, length), and their textual properties such as processed text (Bermingham and Smeaton 2011) and grammatical 
parts. Finally, the best classifier is selected based on its performance on the evaluation set. Performance is measured by the C-statistic measure (AKA, Area Under the Curve - AUC), that is, the capacity of the classifier in discriminating "leak enabling" comments from "non leak enabling" comments.

To enhance system performance and avoid missed leak enabling comments, we tune the classifier prediction-threshold to minimise False Negatives (type II error). In other words, a comment is classified as non leak enabling if the classifier has assigned it with a near-zero probability to be leak enabling, and leak enabling otherwise.

\section{Module 3: UoI}

Module UoI (Users of Interest) is designed to focus on commenters and their on-line user profile, to create a "leaker profile" of each participant. Users' profiles are collected on demand for the set of comments and commenters received from module UoI. On each user, the following profile information is collected: network size - number of friends and number of followers, and privacy setting (whether the user's profile is kept private of public). Potentially additional input information can be collected on each user to measure her FB engagement and online activity.

Similar to CoI, module UoI has two steps: Initialisation and classification. In the initialisation step, a best data-driven classifier is selected and trained on the previously labelled comments to estimate the probability of a commenter to be leak enabling. Here again, the classifier threshold is set to minimise False Negatives. Those identified as UoI are then passed to the module EgoNet.

\section{Module 4: EgoNet}

To rebuild the egocentric network of a UoI we use SocialSpy (Burattin, Cascavilla, and Conti 2015). SocialSpy was developed to retrieve the lists of friends of each UoI, given her publicly available information, such as public friends, pictures, group memberships, and page likes. 
The tool implements four strategies, each using a different type of information from the OSN to rebuild the friends list of a given UoI. The first three strategies are based on liked pages. Statistics show that Facebook Like and Share buttons are used over 22 billion times a day, on approximately 7.5 million Facebook pages (He 2013). Furthermore, like and share information is usually available even for users with high privacy settings.

The fourth strategy exploits likes and comments from the picture of a given user. Although pictures for users are only partially available when a user has high privacy settings, a recent survey shows that many users are unaware of Facebook's privacy options (Consumer Reports Magazine 2012), or too lazy or inexperienced to properly modify them (Madejski, Johnson, and Bellovin 2012). Given that, we expect that this strategy will highlight strong relationships with the UoI and both public and private other users (Jones et al. 2013).

- Strategy 1 exploits like pages of a given profile. Based on the theory of homophily, we can assert that other users who like the same page(s) share common interests with the user of interest, and hence have higher probability of having friendship relationships. Strategy 1 operates as follows. Using the public Facebook pages of the each UoI, Strategy 1 retrieves the list of liked pages left public by the UoI profile. The strategy then retrieves the list of these pages' fans (users who liked these pages). Next, for each fan Strategy 1 queries (via the Mutual Content Page (MCP) (Constine 2010)) whether he is a friend of the User of Interest. The output of this strategy is a list of friends tuples of the format $\left\{U_{o} I_{i}\right.$, friend $\left._{j}\right\}$.

- Strategy 2 is similar to the first strategy, yet differs in the way that probabilities are set: users who share like pages with small sets of fans receive higher probability of sharing friendships with the user of interest. The reasoning here is that these pages are likely to target a narrower interest, and therefore the homophily value of the users who like it is higher.

- Strategy 3 is the opposite of Strategy 2. That is, the probability of sharing mutual 
friends is higher for users who like pages with more fans. The idea behind this strategy is that, fetching like pages from max-to-min number of fans, results in a bigger userpool faster, even when crawling for a single page, in which mutual friendships with the user of interest can be found.

- Strategy 4 exploits public pictures of the given user. The tool then retrieves the list of users who like public pictures of a given UoI, or commented on them. Once the tool obtains the list of users, it checks the friendship between them and the target ID using the MCP.

Among these four strategies, Strategy 4 has proven to be the fastest and with the highest average $(37.12 \%)$ of friends found. Respectively Strategy 1 with an average of $17.5 \%$ of

retrieved friends, Strategy 2 with an average of $17.4 \%$ of retrieved friends and Strategy 3 with an average of $20.8 \%$ of retrieved friends (Burattin, Cascavilla, and Conti 2015). Based on these results we follow Strategy 4 in our experiments. A detailed description of our implementation of this strategy is given in Appendix A.

\section{Module 5: Viz}

Module Viz groups and visualises the egocentric networks obtained in Module EgoNet (visualisation is done via Gephi https://gephi.github.io/). The main goals of this module are to (1) find overlaps between leakers networks, which may provide additional information on the profile of the leakers and the nature of the leak, (2) examine the extent and the potential diffusion of the information leakage, thus the risk associated with the leak, and (3) provide the system user with a network visualisation of these findings.

\section{Summary of DUIL's design}

DUIL is designed to detect information leakers via commenting to on-line news articles with a current focus on FB news pages. To maximise efficiency, the system follows a modular design, 
in which each module is stand-alone and can be removed or replaced by a context-specific module designed for different purposes. Our understanding of these modules, their main roles, and replacability within the overall IS artifact is enhanced through their respective characterisations as information, social, and technology artifacts.

Conceptually, the system operates through three phases: First the search space is setthe list of articles and comments that may contain information leaks (Module 1). Second, the complexity of the analysis is reduced by filtering out noise and thus decreasing the size of the search space (Modules $2 \& 3$ ). Lastly, the user is presented with a basis for detection and risk assessments of the information leak (Modules $4 \& 5$ ). Figure 1 summarise the design of DUIL.

\section{Demonstration}

In this section, pursuant to design science methodology, we present a series of case studies demonstrating the system. We describe the collection of our test data, and show how we used DUIL to obtain identities of leakers and their social networks.

\section{Experimental Design}

We design a full experiment based on real data collected from FB. Use of the system is illustrated through the analysis of articles crawled from FB news pages, in which part of the information is kept private. Specifically, we are interested in articles in which the identity of personnel is withheld. Identity-censorship is one straightforward type of 'material nonpublic information'. Commenters in the know, can share their private information through comments. The experiment utilises the three phases of DUIL.

Module AoI is first used to collect a set of identity-censored news articles, followed by a thread of commenters and comments that can potentially exhibit censorship breaches. Then, we initialise modules CoI and UoI on a subset of the articles collected. The initialisation phase provides us with two classifiers that can be used in real-time. We evaluate the classifiers 


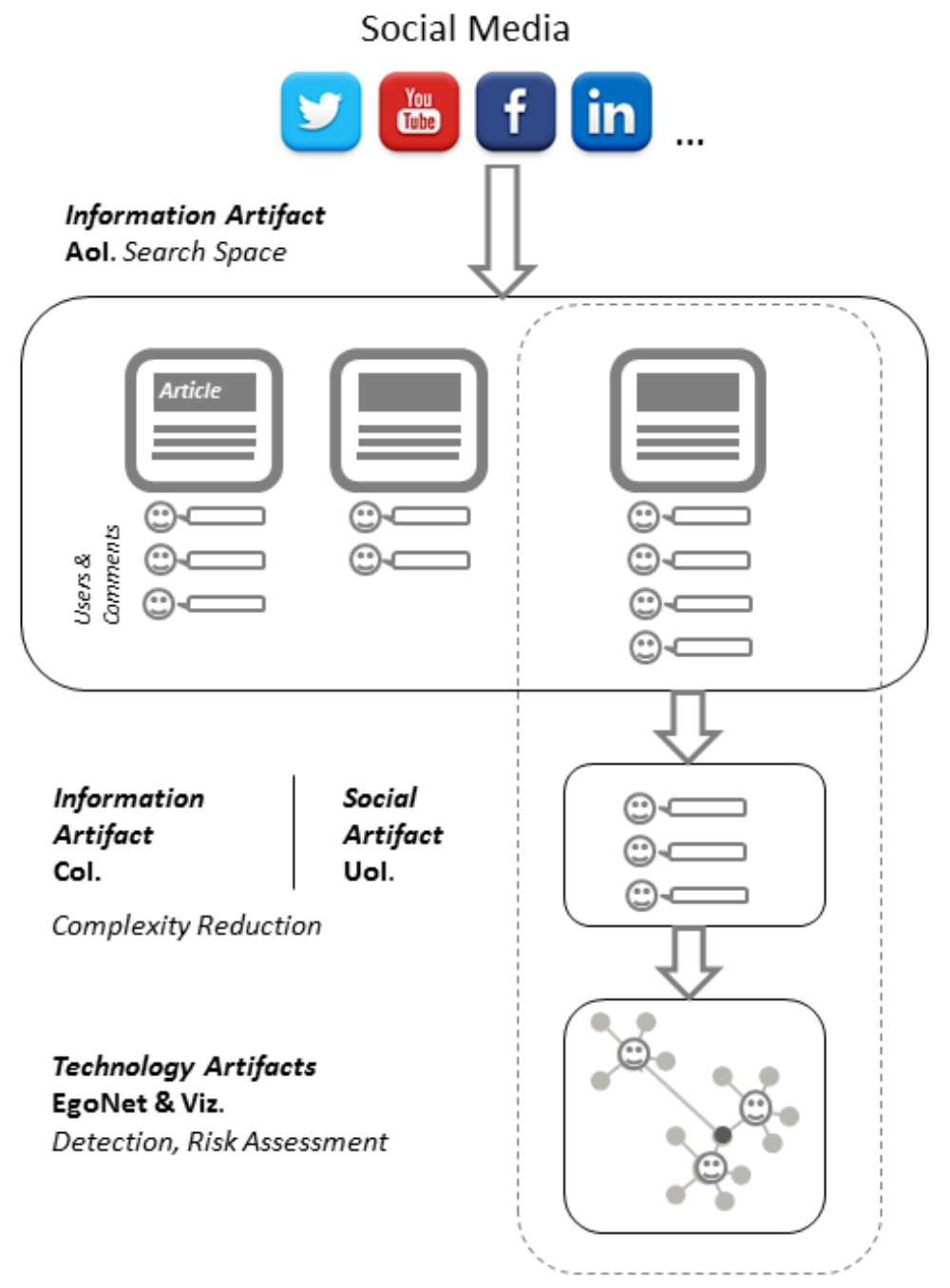

Figure 1: DUIL modules and architecture. 
and report their performance. Finally, we ran modules EgoNet and Viz on 14 selected case studies. In each case we depict a subset of leak-enabling commenters (UoI) and construct the network between them. The case studies illustrate the additional information that the network provides, which includes capturing the leakers and their social networks, and often. though not always, includes identifying the censored personnel.

\section{Description of OSN Data Collected}

We focus on information leakers through comments on Israeli, military-related, news articles published in FB, in which a military personnel name is censored. Censorship in our study is the replacement of a name with a supposedly non-identifying initial (e.g., Corporal S.). Information leakage is detected in the comments published by private users, which leads to the identification of the censored person.

An example of information leakage of interest is presented in Fig. 2. The headline of the news article, as it appears on the FB page of a network news service, is: 'Karakal combat soldier Corporal $S$. who eliminated a terrorist in the course of an incident on the Egyptian border awarded an Honourable citation: Everyone who was with us deserves it, and of course Nathaniel who fell in the battle'. Military information policy dictates that the identity of officers in key positions, or involved in key operations, must not be released to the public. The motivation for this policy is a desire to protect the officer and his or her family from being identified and potentially targeted by hostile persons or forces. In this case, the obfuscated term 'Corporal S.' is identified as the censored element of the news item. A particularly verbose comment associated with the news item states: 'The brave combatant is the daughter of a good friend of mine. Do you know where the combatant comes from? From Elad of course!'. Using DUIL, the readily available identity of that commenter and his FB Friends can lead us to the identity Corporal S. We therefore treat this comment and other similar comments as "leak enabling" comments. A detailed description of the case is discussed in our previous work (BlindedForReview2 2015). 


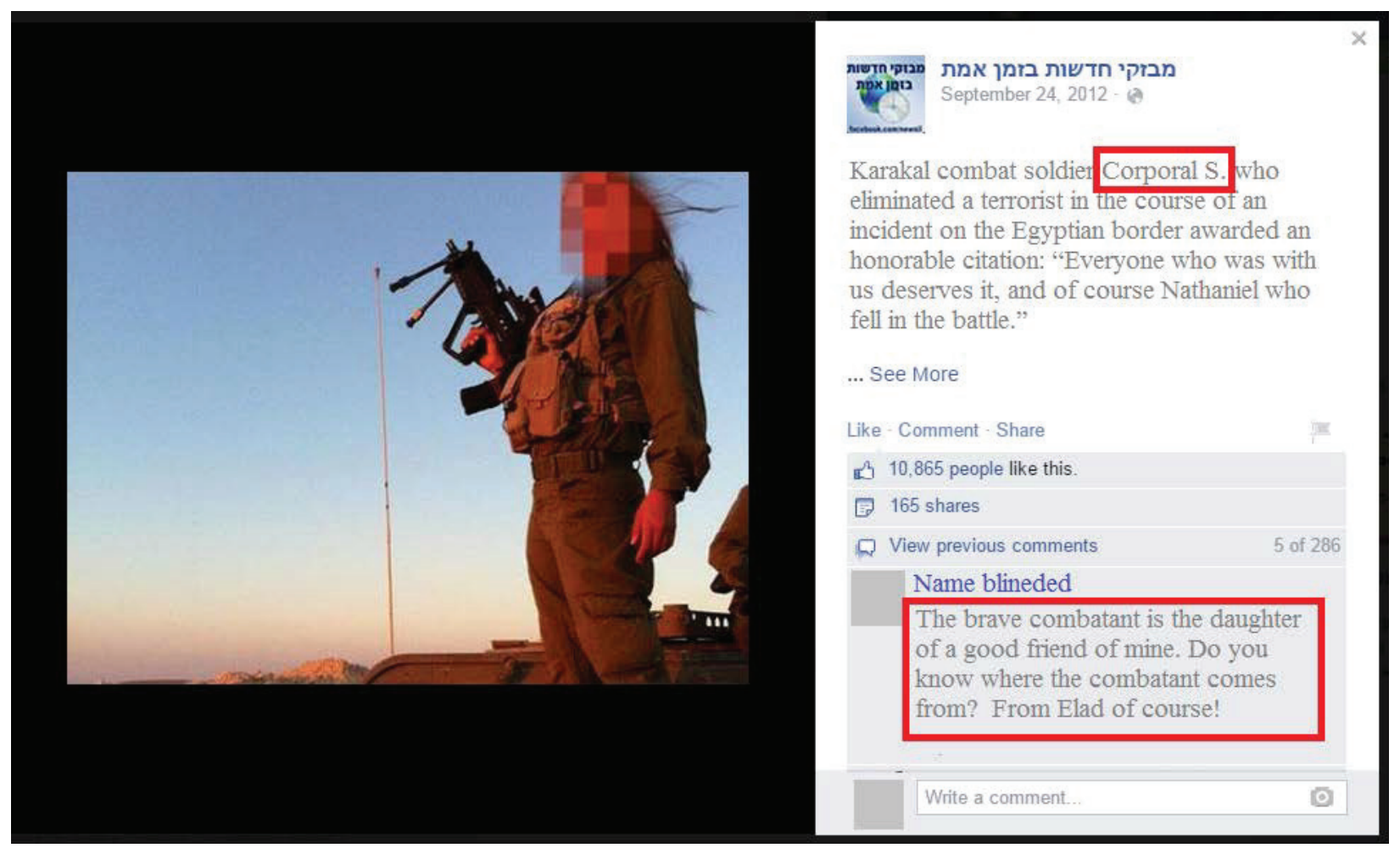

Figure 2: Example of data leakage (translated from Hebrew).

\section{Experimental Results}

We report the results of each system module throughout the course of our experiment.

\section{Module 1: AoI}

AoI screens through a large set of FB news pages, and collects the set of relevant articles. Screening is done via regular expressions defined by the system user.

In our experimental study, we are interested in the set of censored military-related articles, in which the a name of a military personnel is censored. For that, we use a list of regular expression expressions that search for a military rank, followed by an initial letter. For example, the expression '@lieutenant $\backslash \mathbf{c} \backslash$. @' corresponds to the military rank lieutenant followed by his first initial (e.g., lieutenant D.)

The dataset collected contains 48 articles with censored personnel names, with an aggregate total of 3,538 comments. 


\section{Module 2 (CoI) and Module 3 (UoI)}

In this section we present the classifiers constructed for module CoI and UoI, and discuss their performance. We note that the classifiers we chose here are tuned for the data at hand, and might not be optimised to other data-sets. The process of choosing classifiers, however, is data-independent. In the following we repeat the steps of the general process, followed by the specific tuning for our data.

We begin by splitting the set of articles into training, validation, and holdout sets. Independent reviewers are then asked to label comments in the training set and the evaluation set. This labelling is required for system initialisation and evaluation assessment. Ideally, the training and validation sets consist of small samples from the data, as they are labelled manually, yet are big enough to achieve accurate performance.

In our data, for the purpose of performance evaluation, we split the data into training and validation only, each consisting of $50 \%$ of the data. Holdout sample may be defined as comment to all future articles, that are not collected in the current time frame. We then asked four reviewers to classify each comment as either leak enabling or non-leak enabling by reflecting on the following question for each comment: 'Based on this comment, do you believe that the commenter knows the identity of the censored person?'. We then followed a Delphi procedure to achieve agreement among the reviewers. We further asked them to identify the elements of the comment that caused them to reach their conclusion.

Out of the 3,538 comments collected on 48 articles by the AoI module, the reviewers labelled $149(4.21 \%)$ as leak enabling comments. Interestingly, these comments are spread out through $75 \%$ (36) of the articles. A summary of the comments classification is presented in Table 1.

After the data are labelled, different classification algorithms are trained on the data. Classification algorithms include but not limited to logistic regression, SVM, classification tree, and classification forest. The methods are evaluated based on their ability in capturing the relationship between leak enabling comments and features of the comments, using the 
Table 1: Frequency (f) and proportion (\%) of comments' classes

\begin{tabular}{lll}
\hline Comment Type & $\mathbf{f}$ & $\mathbf{\%}$ \\
\hline Leakage comments & 149 & $4.21 \%$ \\
Non-Leakage comments & 3389 & $95.79 \%$ \\
Total comments & 3538 & $100 \%$ \\
\hline
\end{tabular}

C-statistic measure (AKA, Area Under the Curve - AUC). The features used in our current system are divided into three families, each used by the classifiers solely and in combination with other families. The first family contains general quantitative characteristics of the comments, such as its order, its length, its popularity (as obtained from FB), and the present of semiotics in the comment (smiles, winks, etc.). The second family of features is the textual features extracted via the comment level sentiment analysis in Bermingham and Smeaton (2011) on both processed text (lemmatized text, after the removal of stop words), and grammatical parts such as lexical part-of-speech, gender, tense, number (singular, plural), and person (1st, 2nd, 3rd). The last family contains the main elements mentioned by the reviewers and are data-specific. In our context, this family include the repetition of censored information within the comment, expression of affection, mention of a location not mentioned in the article, or personal experience related to the content of the article.

In our dataset, out of the models we examined, the best performance on the evaluation set, using cross validation, was that given by a logistic regression, using features from the first and the third families of features. The output of the model and the features selected are given in Table 2. The C-statistic of the model, that is, its capacity in discriminating leak enabling comments from non leak enabling comments, is $81 \%$. The performance on the model is summarised in the Receiver-Operating characteristic (ROC) Curves in Fig. 3. The ROC Curve depicts the trade-off between False Positive Rate and False Negative Rate for different classification thresholds: the probability cutoff for classifying comments as leak-enabling.

To minimise missed leak-enabling comments, our system next selects the threshold that minimises False Negatives (type II error), assuring that comments are only classified as non 
leak enabling if the classifier has assigned it with a near-zero probability, and leak enabling else-wise.

For our data, as observed by the ROC curve, this threshold equals 0.75 . Under this threshold approximately $26 \%$ of the comments can be ruled out as non leak enabling, without significantly increasing the model's False Negatives (less than 5\% error). This threshold is marked with dash line in Fig. 3.

A similar process is carried for the UoI module. For our data, the best classifier achieved for this module is the logistic regression given in Table 3. The C-statistic of the model is $60 \%$. Given the fairly low C-statistic, the low model coefficients and their (in)significance, we conclude that UoI module in our case study does not provide additional information on top of the CoI module. Reasons for this can be attributed to data size and information available for each profile. Note that this result only holds for this specific set of articles. However UoI might be useful for other data-sets or detection purposes.

Table 2: CoI logistic model

\begin{tabular}{lll}
\hline Predictor & Estimate & P-value \\
\hline (Intercept) & -3.77 & $\sim 0$ \\
Comment Popularity & 0.00 & $\sim 0$ \\
Repetition of censored information & 2.28 & $\sim 0$ \\
Mentions of location & 0.84 & 0.15 \\
Mentions of personal experiences & 1.41 & $\sim 0$ \\
Semiotics (smiles,winks, etc.) & 0.66 & $\sim 0$ \\
Expressions of affection & 2.40 & $\sim 0$ \\
\hline
\end{tabular}

Table 3: UoI logistic model

\begin{tabular}{lll}
\hline Predictor & Estimate & P-value \\
\hline (Intercept) & -0.05 & $\sim 0$ \\
Network Size & 0.0007 & 0.008 \\
Followers & 0.006 & 0.42 \\
Privacy Setting & -0.51 & 0.42 \\
\hline
\end{tabular}




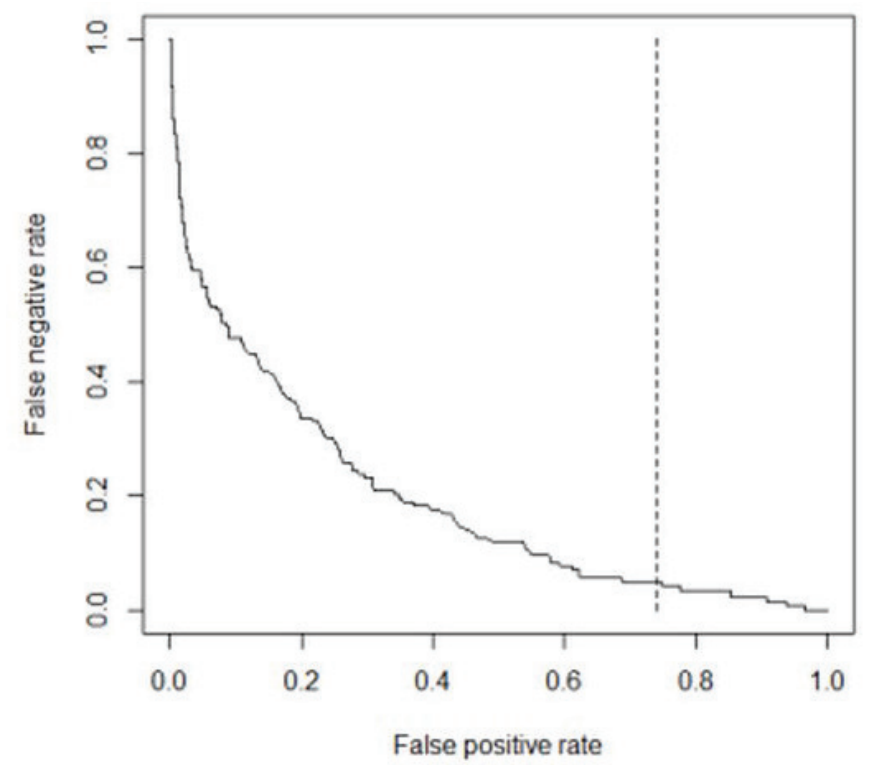

Figure 3: ROC curves of the logistic models.

\section{Module 4 (EgoNet) and Module 5 (Viz)}

We run modules EgoNet and Viz on 14 articles randomly selected from our output of AoI. For each article EgoNet and Viz are run on all users screened through module UoI. EgoNet and Viz (re)build and visualise the friends ego networks of the list of UoI, including mutual friends.

Fig. 4 illustrate two of the more interesting cases. The black nodes in the figure are the UoI. UoI are surrounded by their friends, some of which are common between them.

In the first case (Fig. 4(a)) we can see that four UoI are not FB-friends: there is no direct connection between them. They share a single friend, which we later found to be the censored person from the article. In the second example, plotted in Fig. 4(b), it is observed that all five UoI are strongly connected, and share multiple friends.

Due to the nature of the articles and the data chosen, that is, identity-censored article, the ego networks may provide us with additional useful information: the identity of the censored personnel. This information will become immediately available when the leakers are FB-friends with this person. In the two examples we present, this is the case. In each panel of figure 4, a single white circular node was manually confirmed to be the censored 
personnel. Confirming the censored person identity was done thanks to finding the blurred picture from the article, unblurred in the users' profile.

Out of the 14 experiments, we were able to identify the censored personnel in four cases. In each of the other 10 cases, a network was constructed, and mutual friends of UoI were found in 8 of the cases. However, we could not verify nor refute their link to the censored personnel.

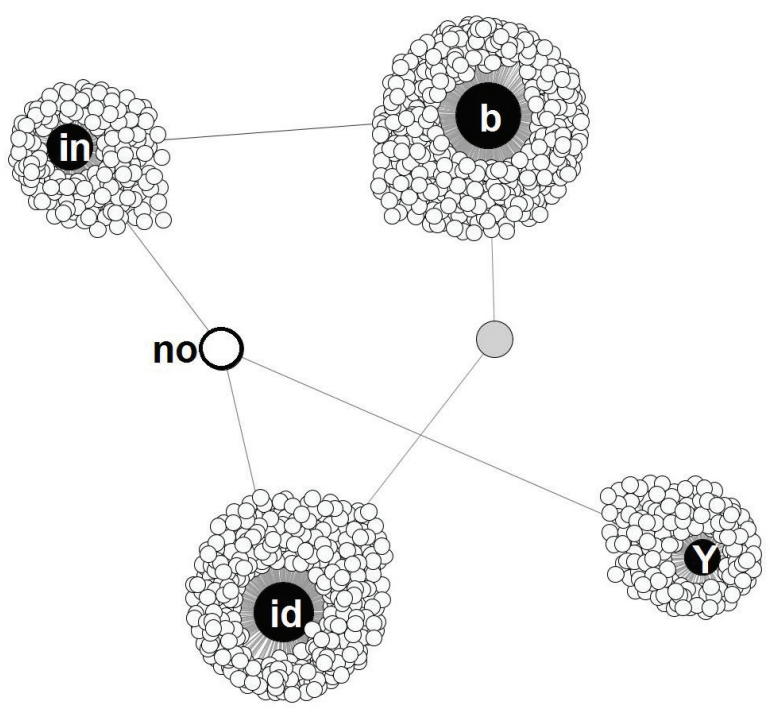

(a)

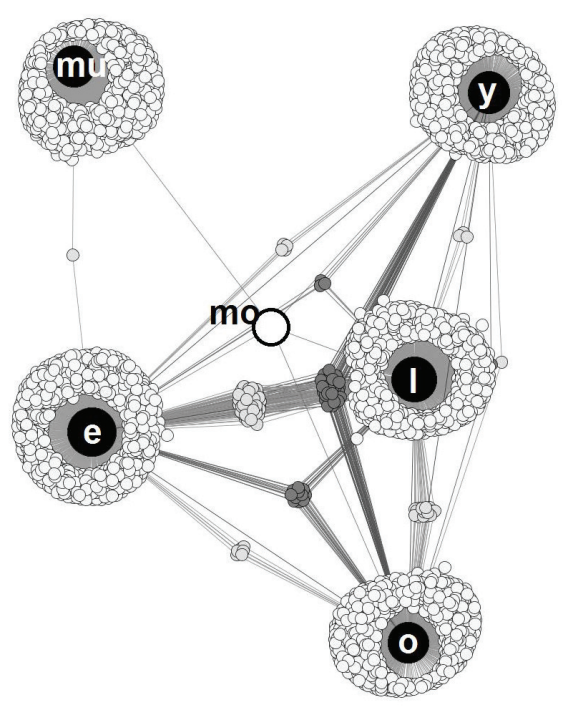

(b)

Figure 4: Networks generated by module Viz.

\section{Discussion and Conclusion}

DUIL is a new type of system that can be considered as a member of the superclass of Social OSINT systems, a form of cyber-threat intelligence system, which are growing in importance (Casanovas 2017; Bowman et al. 2016; Jasper 2017; Nunamaker et al. 2017).

The combination of comment and user mining with risk analysis, and of social network visualisation for risk signalling, is the main system-based contribution. This produces synergies in terms of new analytical capabilities. Such analytical requirements are a moving 
target which makes the decisions to divide components all the more important.

To provide a more granular discussion we frame DUIL as an information systems artefact as presented by Lee, Thomas, and Baskerville (2015). There are few salient examples of related work that has taken a design science approach focused on the IS artifact rather than the IT artifact. Huhtamäki, Russell, and Sill (2016) perform ecosystem analytics by integrating a technology artifact with other artifacts to perform visual network analytics. Both Spagnoletti, Resca, and Sæbø (2015) and Wakefield and Wakefield (2016) tackle social media technologies as a three-dimensional information systems artifact comprised of technical, informational, and social sub-artifacts.

Following Lee, Thomas, and Baskerville (2015), we divide this part of our discussion into three, covering:

1. the specific IT artifacts developed,

2. the information artifacts detected and collected, and

3. the social artifact that influences, in our case, both the IT and information artifacts.

All three taken together comprise the IS artifact in which IT artifacts comes together with other artifacts that are not strictly IT so that "they ultimately serve to solve a problem or achieve a goal for individuals, groups, organizations, societies, or other social units" (pg 6).

AoI and CoI are information artifacts. The three data tables collected by AoI together comprise an information artifact which serves a central purpose in the overall IS artifact. This corresponds to the definition proposed by Lee, Thomas, and Baskerville (2015) wherein the artifact (a) consists of an instantiation of information and (b) it is generated by a human initiating use of a computer program in this case a FB crawler and filtering mechanism. Similarly, CoI is an information artifact. Corresponding to the definition wherein the artifact (a) consists of an instantiation of information and (b) it is generated by direct human action - in this case expert classification, combined with initiating use of a computer program in this case a classifier mechanism. 
Such instantiations of information will recur repeatedly throughout the life and use of the overall system. Furthermore, the design of the overall system is such at that the information artifacts we use in the current study can be replaced, without loss of generality, by information collected from other media sources and processed by different classifiers, thus strengthening the appropriateness of the information artifact definition within the IS artifact framework. In other words, it would be misleading to consider the AoI or UoI as technology artifact, as it is not a technology making the decisive contribution but rather the information gathered at this stage.

UoI is a social artifact. The UoI module meets the frameworks definition of a social artifact in that it reflects relationships or interactions between or among individuals, involving the social and not just the individual. This characterization is seen in the social-behavioral data collected and processed by this artifact which includes number of friends and FB user activity.

EgoNet and Viz are technology artifacts. In EgoNet and Viz we have instantiations of pure IT artifacts as defined by the IS artifact framework. Both are human-created tools used to solve a problem or achieve a goal. In the case of the former the goal is to (re)build a previously unknown egocentric network, and in the case of the latter provide visualization.

Taken together, and given the interactions and interdependencies between the two information artifacts, one social artifact, and two IT artifacts, we have a prototypical example of an IS artifact as per the Lee, Thomas, and Baskerville (2015) framework.

Other than defining three of many possible artifact types, Lee, Thomas, and Baskerville (2015) provide little guidance on determining artifact categorization and we note that such categorization may not be obvious. For example, one might argue that a given artifact such as our AoI is a technology artifact rather than an information artifact when observing that it is a software technology enabling automated identification of the relevant articles. However in making this assessment we look to the main contribution of the artifact to the design which, in the case of AoI is not in the technological way in which the information was 
achieved, but in the essence of having collected the information itself.

Based on our experience we have found that the concept of artifact contribution can be essential in determining whether an artifact should be viewed as social, information, or technology. We emphasize that the distinction is not always clear cut and requires careful consideration. Table 4 summarizes.

The Lee et al approach to IS artifacts is not without controversy. Juhani (2016) points out, that Lee et al. simply interpret IT artifacts as purely technical ones which he considers a potential shortcoming. We have found, however, that this narrow definition when used alongside the complementary social and information artifacts, enables a rich and precise descriptive and analytical discourse. Amongst other critiques, Juhani (2016) questions how Lee, Thomas, and Baskerville (2015) might associate their work with extant approaches to design science as this is not explicitly addressed in their work. We believe that our work has provided an initial answer to this question, and we have found and demonstrated that the Lee at al. framework can coexist quite nicely with traditional design science. Finally, Juhani (2016) questions how design science research would make direct contributions to the non-IT artifacts in the Lee et al. framework. We have found that the characterization of certain artifacts as social or information eases their placement and analysis within an overall IS design project. Rather than opening an unmanageable distance between the artifact and DSR, it forces us to think in terms of the actual contribution of each artifact to the IS rather than limiting our assessment to technological contribution.

\section{Meeting the Objectives and Assessing Contribution}

Our system design goals specified three sets of objectives: modularity; automation, and accuracy; visualization of leakers' social networks.

The modularity goal is achieved by the choice of architecture. Most important in this respect is the ability to replace Module 1: AoI (Articles of Interest) and Module 2: CoI (Comments of Interest) with alternatives that can process other forms of social media. Sep- 
Table 4: Determining artifact type

\begin{tabular}{|c|c|c|}
\hline Artifact & Type & Determining Contribution \\
\hline $\begin{array}{l}\text { Articles of Inter- } \\
\text { est \& Comments } \\
\text { of Interest }\end{array}$ & Information & $\begin{array}{l}\text { Q. Does the fact that a technology is used to gather the } \\
\text { information not make these technology artifacts? } \\
\text { A. No, as the technological implementation is not what } \\
\text { contributes to the system design., There are multiple, per- } \\
\text { haps equally valid, technological approaches. The contri- } \\
\text { bution of the artifact lies in the information, not the tech- } \\
\text { nology.,Different information sources plugged into this } \\
\text { artifact might require alternative technologies. }\end{array}$ \\
\hline Users of Interest & Social & $\begin{array}{l}\text { Q. The user data is provided by FB which is a technol- } \\
\text { ogy implementing a social network, so perhaps this is a } \\
\text { technology artifact? } \\
\text { A. No, as the technological implementation is not what } \\
\text { contributes to the system design. There are multiple pos- } \\
\text { sible social networks that might be analyzed as part of the } \\
\text { information system. The contribution of this artifact to } \\
\text { the overall information system lies in the social structures } \\
\text { provided, not in the technology that supports it.,Different } \\
\text { social networks plugged into this artifact might require } \\
\text { alternative technologies, and provide different social in- } \\
\text { sights. }\end{array}$ \\
\hline EgoNet & Technology & $\begin{array}{l}\text { Q. This artifact is meant to provide information about } \\
\text { network structures, so perhaps this is an information ar- } \\
\text { tifact? } \\
\text { A. No, as the contribution of this artifact to the system } \\
\text { was to enable the creation of the required network struc- } \\
\text { tures where no such capability previously existed. }\end{array}$ \\
\hline Viz & Technology & $\begin{array}{l}\text { Q. This artifact presents social structures in a visual man- } \\
\text { ner so perhaps it is a social artifact not a technology ar- } \\
\text { tifact? } \\
\text { A. No, as the primary contribution of this artifact is } \\
\text { to determine a visually effective way to present network } \\
\text { data. This contribution is technological in can be repur- } \\
\text { posed for use in different network domains. }\end{array}$ \\
\hline
\end{tabular}


arating out Module 3: UoI (Users of Interest) further extends the desired flexibility for different OSN structures.

Automation and per-module accuracy goals have been partially achieved at the proofof-concept level as demonstrated by the experiments. Accuracy is measured via the AUC measure for the statistical module, yet cannot be measured for the Viz module, as we later discuss in the limitation section. Further experimentation will be required in these areas.

The visualization of leakers' social networks goal has been achieved as illustrated by the experiments and accompanying graphs.

Beyond meeting the objects set at the outset of the system design process, we briefly address DSR knowledge contribution as discussed in Gregor and Hevner (2013). Their framework assesses contribution on the axes of x:problem maturity and y:solution maturity. Scaling regions of high and low for each axis gives the four quadrants of:

1. Routine design (high,high) applying known solutions to known problems, resulting in no major knowledge contribution;

2. Exaptation (low, high) extending known solutions to new problems, resulting in research and knowledge contributions;

3. Improvement (high, low) developing new solutions to known problems, resulting in research and knowledge contributions; and

4. Invention (low, low) inventing new solutions for new problems, resulting in research and knowledge contributions.

Identifying leakers of material nonpublic information is not a new problem, as we see from the analogy to insider trading and organizational information leakage described in our introduction. However the shift of this problem from inside the organization to the broad context of social media has introduced significant new complexities to the problem, changing important problem characteristics particularly with regards to scale and scope. Therefore 


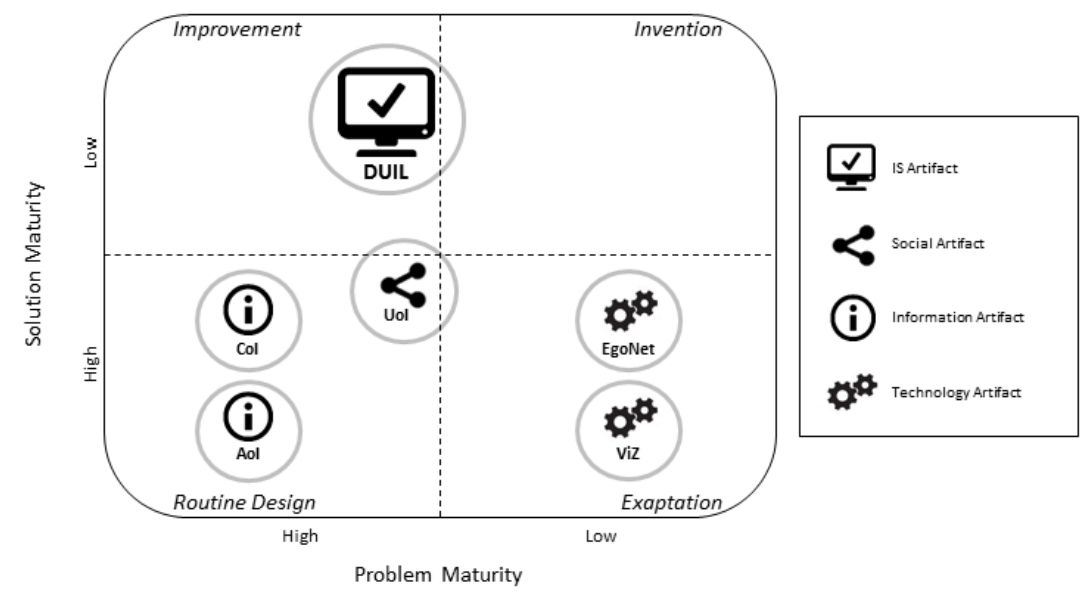

Figure 5: DSR knowledge contribution framework with multiple artifacts (after Gregor and Hevner (2013)).

on the problem maturity axis DUIL would score a mid-range value rather than a clear high expected of an improvement and clear low expected of an invention. On the solution maturity axis the DSR contribution of DUIL is clearly in the low range owing to the novelty of the approach and previously un-attempted combination of artifacts in a complex information system artifact. Therefore based on the criteria set out by Gregor and Hevner (2013) our DSR contribution lies in the upper right of the improvement quadrant extending slightly into the invention quadrant. We present this graphically in Figure 5 modelled after the Gregor and Hevner framework.

In testing their framework Gregor and Hevner (2013) presented a table of 13 design science articles classified by knowledge contribution type. They document the classification of a single contribution per article, into a distinct quadrant of the grid. We suggest that when following the multi-artifact approach to IS artifacts, research value can be revealed by mapping the contribution of each component artifact when presenting an information system. We therefore have enhanced the original DSR contribution framework diagram to show the positioning of the sub-artifacts that comprise the DUIL information system artifact. Each artifact type is represented by a different symbol.

The two information artifacts, AoI and CoI appear in the routine design quadrant. These 
artifacts use a combination of human intervention and text processing that are well-known in the art and applied to many text classification problems. Therefore they rank high on both axes of problem maturity and solution maturity. This means that taken on their own these artifacts provide no research and knowledge contribution.

The two technology artifacts, EgoNet and Viz, appear in the exaptation quadrant. Here we have examples of two technologies that have been used successfully in other domains, being re-purposed to solve a new problem. Therefore they rank high on the solution maturity axis and low on the problem maturity axis.

The social artifact, UoI, appears straddling the routine design and exaptation quadrants, with a slight advance upward into the improvement and invention quadrants. This indicates a solution design that has elements of routine use (tracing the known social networks of commenters, for example), elements of exaptation (finding new uses of the social structures for leak containment), while being applied to a newly complex instantiation of an existing problem.

Following the DSR knowledge contribution guidelines and enhancing the graphical presentation for multiple artifacts, helps to effectively express the knowledge contributions of DUIL.

\section{Limitations}

Though DUIL reaches its design and security goals, there are some unavoidable limitations and shortcomings. The main limitation of DUIL is reliance on human intervention for initialization, thus can be considered a semi-automated information system. Specifically, four actions are done manually:

1. In module AoI, system user defines the context, formulated as a list of regular expressions. Alternatively, page administrator can flag articles that need to be monitored for potential leakers. 
2. Initialization of CoI involves experts annotation of a sufficient training set. Expert annotation is customary in classifying UGC.

3. In modules CoI and UoI, system users can, but are not obligated to, control the list of data features and classifiers, according to their domain knowledge and experience.

4. Modules EgoNet and Viz provide and overview of the potential leak and involved leakers, yet it is up to the user to carry on further analysis of the threat.

The second limitation of the system relates to the expert annotation, which is needed since no ground truth is available. In practice a comment can be misclassified by reviewers as leak enabling, while in fact it is not.

The third limitation stems from the use of statistical models in a Swiss-Cheese fashion. Modules AoI (if pre-post labeling was chosen), CoI, and UoI may introduce some statistical errors (false positives and false negatives) into the system. These error are carried on through the modular design, resulting in potentially increased error rate.

\section{Future Research}

Given the great popularity of OSN, they have become one of the most common means to share and discuss information, including news articles. We find that comments and commenters' OSN leak information, originally withheld in news articles. To underline the importance of this issue, in this paper we present DUIL, a system we designed and implemented to analyze news comments in order to detect information leakers and assess the risk associated with such leaks. We ran real-data experiment on military news articles, the results underlines the effectiveness of our approach in finding leakers amongst UoI and (re)building their social network. Moreover, our tool was able to de-censor the information in some of the articles.

This study, aside from the primary contribution of knowledge created by our specific IS artifact, has secondary theoretical contributions. It provides a crisp illustration of how the IS artifact framework espoused by Lee, Thomas, and Baskerville (2015) can effectively aid 
in the description and analysis of a complex system, and has extended the use of Gregor and Hevner (2013)'s DSR knowledge contribution framework.

Detection of leaked information, identification of a leaker, and quantification of the impact of a leak are three distinct yet interrelated challenges faced by organizations in the age of OSN. DUIL was designed and implemented with the holistic view of addressing all three of these challenges. Through DUIL and the systems that follow it, organizations will be able to assess and address their exposure to the risks of information leakage. With the inherent structure of OSNs turning more users into bearers of material non-public information, addressing these challenges will continue to grow in importance.

\section{Acknowledgements}

Temporarly removed for blind review.

\section{References}

AGICHTEIN, E. et al. (2008). "Finding high-quality content in social media". In: Proceedings of the 2008 international conference on web search and data mining. ACM, pp. 183-194. BERMingham, A. and A. SMEATON (2011). "On Using Twitter to Monitor Political Sentiment and Predict Election Results". In: Proceedings of the Workshop on Sentiment Analysis where AI meets Psychology (SAAIP 2011). Chiang Mai, Thailand: Asian Federation of Natural Language Processing, pp. 2-10.

BISHOP, M. and C. GATES (2008). "Defining the Insider Threat". In: Proceedings of the 4th Annual Workshop on Cyber Security and Information Intelligence Research: Developing Strategies to Meet the Cyber Security and Information Intelligence Challenges Ahead. Ed. by S. FREDERICK et al. CSIIRW '08. ACM, 15:1-15:3.

BLINDEDFORREVIEW2 (2015). "Blinded For Review". In: 
BOWMAN, E. K. et al. (2016). "Content-Based Multimedia Analytics: Rethinking the Speed and Accuracy of Information Retrieval for Threat Detection". In: STO Meeting. NATO Science and Technology Organization. Vol. 18. NATO, pp. 1-10. (Visited on 05/22/2017).

BURATTIN, A, G CASCAVILlA, and M CONTI (2015). "SocialSpy: Browsing (Supposedly) Hidden Information in Online Social Networks". In: Lecture Notes in Computer Science. Springer International Publishing, pp. 83-99.

CASANOVAS, P. (2017). "Cyber Warfare and Organised Crime. A Regulatory Model and Meta-Model for Open Source Intelligence (OSINT)". In: Ethics and Policies for Cyber Operations. Springer, pp. 139-167.

CASCAVILLA, G. et al. (2015). "Revealing Censored Information Through Comments and Commenters in Online Social Networks". In: Proceedings of the 2015 IEEE/ACM International Conference on Advances in Social Networks Analysis and Mining, ASONAM 2015, Paris, France, August 25 - 28, 2015, pp. 675-680. URL: http://doi.acm.org/10. $1145 / 2808797.2809290$.

CLAUDIO, C.-R. (2009). "Modelling Deterrence in Cyberia". In: NATO Science for Peace and Security Series - E: Human and Societal Dynamics 59.Modelling Cyber Security: Approaches, Methodology, Strategies, pp. 125-131.

COnStine, J. (2010). Facebook Announces Friendship Pages That Show Friends' Mutual Content. http://www . insidefacebook. com/2010/10/28/friendship-pages-mutualcontent. (Accessed on 2016-11-24).

GREGOR, S. and A. R. HEVNER (2013). "Positioning and Presenting Design Science Research for Maximum Impact". In: MIS Quarterly 37.2, pp. 337-356.

HE, R. C. (2013). Facebook developers page - Introducing new Like and Share buttons. https: //developers.facebook.com/blog/post/2013/11/06/introducing-new-like-andshare-buttons. (Accessed on 2016-11-24).

HEVNER, A. and S. CHATTERJEE (2010). Design Research in Information Systems: Theory and Practice. 1st. Springer US. 
HEVNER, A. R. et al. (2004). "Design Science in Information Systems Research". In: Mis Quarterly 28.1, pp. 75-105.

HUDDART, S. J. and B. KE (2007). "Information Asymmetry and Cross-sectional Variation in Insider Trading*”. In: Contemporary Accounting Research 24.1, pp. 195-232.

HUhtAmÄKI, J., M. G. RUSSELL, and K. SILL (2016). "Processing Data For Visual Network Analysis". In: Visual Analytics for Management: Translational Science and Applications in Practice. Ed. by B. ELLIOT and C. SACHA. Oxford: Routledge. Chap. 5.

IIVARI, J. (2014). "Distinguishing and contrasting two strategies for design science research". In: European Journal of Information Systems 24.1, pp. 107-115.

IM, G. P. and R. L. BASKERVILLE (2005). "A longitudinal study of information system threat categories: the enduring problem of human error". In: ACM SIGMIS Database 36.4, pp. 68-79.

INC., F. (2015). Facebook Reports Third Quarter 2015 Results. http://investor.fb.com/ releasedetail. cfm?ReleaseID=940609s. (Accessed on 2016-11-24).

JASPER, S. E. (2017). "US Cyber Threat Intelligence Sharing Frameworks". In: International Journal of Intelligence and CounterIntelligence 30.1, pp. 53-65.

JONES, J. J. et al. (2013). "Inferring Tie Strength from Online Directed Behavior". In: PLOS ONE 8.1, pp. 1-6.

JUHANI, I. (2016). "Information system artefact or information system application: that is the question". In: Information Systems Journal, n/a-n/a.

KANDIAS, M. et al. (2013). "Proactive Insider Threat Detection Through Social Media: The YouTube Case". In: Proceedings of the 12th ACM Workshop on Workshop on Privacy in the Electronic Society. WPES '13. Berlin, Germany: ACM, pp. 261-266.

KARSCH, M. (1984). "The Insider Trading Sanctions Act: Incorporating a Market Information Definition". In: Journal of Comparative Business and Capital Market Law 6.3, pp. 283-305. 
LEE, A. S., M. THOMAS, and R. L. BASKERVILLE (2015). "Going back to basics in design science: from the information technology artifact to the information systems artifact". In: Information Systems Journal 25.1, pp. 5-21.

LYYTINEN, K. and J. L. KING (2004). "Nothing at the center?: Academic legitimacy in the information systems field". In: Journal of the Association for Information Systems 5.6, pp. 220-246.

MADEJSKI, M., M. JOHNSON, and S. M. BELLOVIN (2012). "A study of privacy settings errors in an online social network". In: 2012 IEEE International Conference on Pervasive Computing and Communications Workshops. IEEE, pp. 340-345.

MAGAZINE, C. R. (2012). Facebook \& your privacy. http://www . consumerreports .org/ cro/magazine/2012/06/facebook-your-privacy. (Accessed on 2016-11-24).

MARKUS, L. M., A. MAJCHRZAK, and L. GASSER (2002). "A Design Theory for Systems That Support Emergent Knowledge Processes". In: MIS Quarterly 26.3, pp. 179-212.

NUNAMAKER, J. F. et al. (2017). "Creating High-Value Real-World Impact through Systematic Programs of Research." In: MIS Quarterly 41.2.

ORLIKOWSKI, W. J. and C. S. IACONO (2001). "Research Commentary: Desperately Seeking the IT in IT ResearchA Call to Theorizing the IT Artifact". In: Information Systems Research 12.2, pp. 121-134.

ORLIKOWSKI, W. J. and C. S. IACONO (2006). "The Artifact Redux: Further Reflections on the ITin IT Research". In: Information Systems: The State of the Field. Ed. by J. L. KING and K. LYYTINEN. John Wiley \& Sons. Chap. 12, pp. 287-292.

PEFFERS, K. et al. (2007). "A Design Science Research Methodology for Information Systems Research". In: J. Manage. Inf. Syst. 24.3, pp. 45-77.

REASON, J. (1990). "The Contribution of Latent Human Failures to the Breakdown of Complex Systems". In: Philosophical Transactions of the Royal Society of London B: Biological Sciences 327.1241, pp. 475-484. 
SCHWARTZ, D. G. (2014). "Research CommentaryThe Disciplines of Information: Lessons from the History of the Discipline of Medicine". In: Information Systems Research 25.2, pp. 205-221.

SEIN, M. K. et al. (2011). "Action Design Research". In: MIS Quarterly 35.1, pp. 37-56.

SPAGNOLETTI, P., A. RESCA, and Ø. SÆBØ (2015). "Design for Social Media Engagement: Insights from elderly care assistance". In: The Journal of Strategic Information Systems 24.2, pp. 128-145.

STRUDLER, A. and E. ORTS (1999). "Moral Principle in the Law of Insider Trading". In: 78, pp. $375-438$.

TWYMAN, N. W. et al. (2014). "Autonomous Scientifically Controlled Screening Systems for Detecting Information Purposely Concealed by Individuals". In: Journal of Management Information Systems 31.3, pp. 106-137.

WAKEFIELD, R. and K. WAKEFIELD (2016). "Social media network behavior: A study of user passion and affect". In: The Journal of Strategic Information Systems 25.2, pp. 140156.

WARKENTIN, M. and R. WILLISON (2009). "Behavioral and policy issues in information systems security: the insider threat". In: European Journal of Information Systems 18.2, pp. 101-105.

YAHAV, I., D. G. SCHWARTZ, and G. SILVERMAN (2014). "Detecting unintentional information leakage in social media news comments". In: Proceedings of the 15th IEEE International Conference on Information Reuse and Integration, IRI 2014, Redwood City, CA, USA, August 13-15, 2014, pp. 74-79. URL: https://doi.org/10.1109/IRI. 2014. 7051874 .

ZHOU, L. et al. (2004). "A Comparison of Classification Methods for Predicting Deception in Computer-Mediated Communication". In: Journal of Management Information Systems 20.4, pp. 139-165. 


\section{Appendix A: Implementation of EgoNet Strategy 4}

1. EgoNet receives as input the list of UoI.

2. For each user in the list, EgoNet obtains the set of publicly available albums, and public pictures within these albums (line 1 of Algorithm 1).

3. For each picture the tool collects the identities of users who commented or liked the picture (line 4; the users who left a comment or pressed the Like button are defined respectively in the algorithm with $U_{i}^{c}$ and $U_{i}^{l}$.)

4. Using MCP (Constine 2010), EgoNet

- checks whether the UoI and a given user are friends 'Facebook friends since [date]' (line 7);

- if yes, retrieves the list of common friends (line 8).

5. Lastly, EgoNet, returns the list of the 'Friends Found' (line 9).

Algorithm 1 implements the steps taken for each user in the UoI set. 


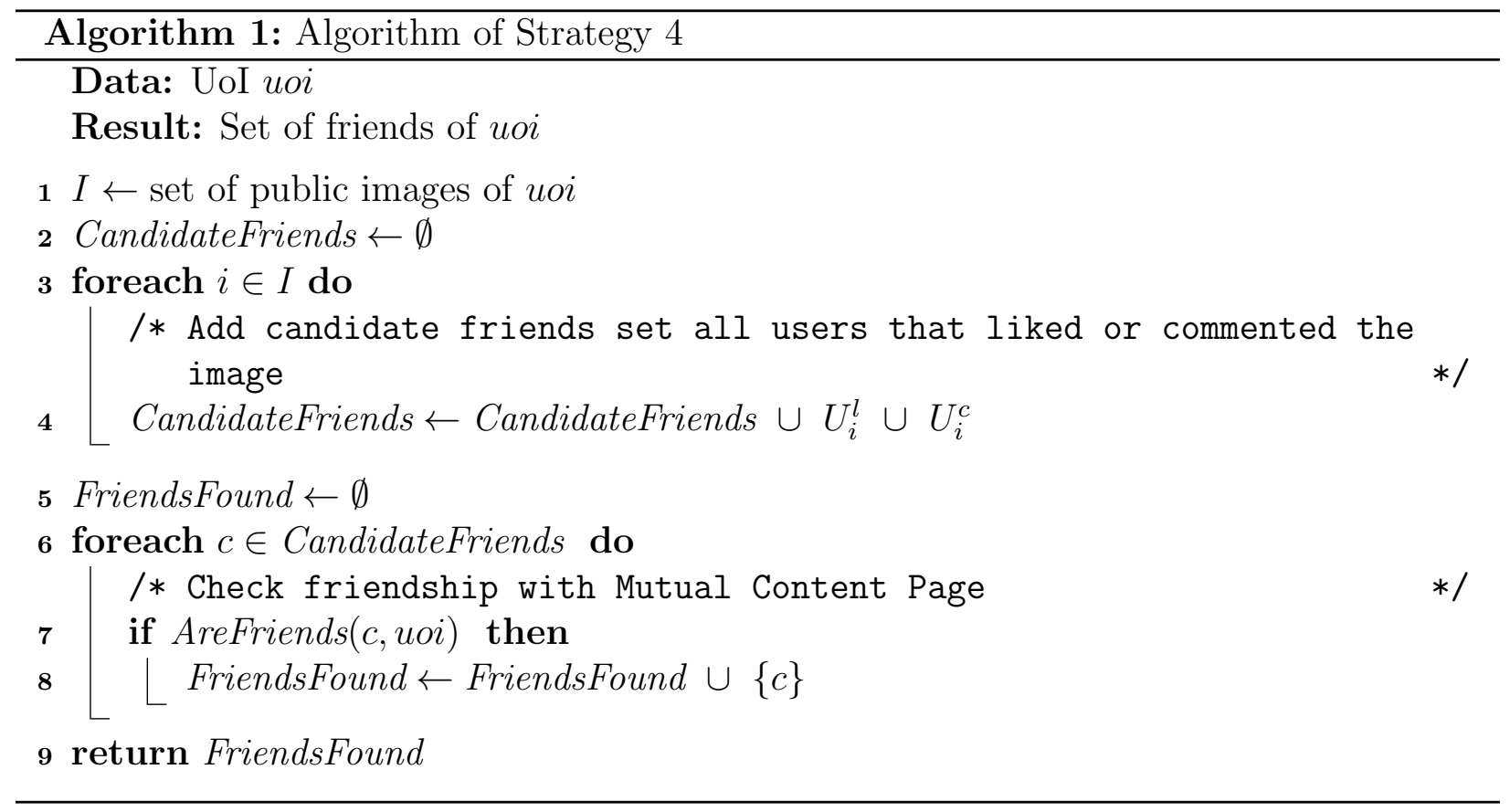

